\title{
Pé em risco aumentado de ulceração em doentes com diabetes mellitus tipo 2
}

António Macedo,* Carla Campos, ** Joana Correia,* Paula Gomes***

\section{RESUMO}

Objectivos: Com este estudo pretendeu-se determinar a prevalência de pé em risco aumentado de ulceração em doentes diabéticos tipo 2 no concelho de Matosinhos, utilizando a classificação do International Working Group Diabetic Foot (IWGDF) e sua correlação com algumas variáveis da população.

Tipo de Estudo: observacional analítico transversal.

Local: Três centros de saúde (CS) do Concelho de Matosinhos.

População: Doentes com Diabetes Mellitus tipo 2, portadores de Guia do Diabético e residentes em Portugal.

Métodos: Obtiveram-se 237 participantes pela técnica de amostragem aleatória simples com reposição. Os dados foram obtidos por entrevista presencial, exame objectivo e análise de processos. Colheram-se dados gerais da amostra bem como Índice de Massa Corporal, período desde diagnóstico e controlo glicémico. Foi avaliada presença de vasculopatia e neuropatia periférica, de modo a agrupar os doentes pela classificação do IWGDF.

Resultados: Dos 237 participantes, $46 \%$ eram do sexo masculino com média de idades de 63 anos. A prevalência de pé em risco aumentado de ulceração encontrada foi de 18,1\% encontrando-se associação com a situação de reformado.

Conclusões: Este estudo revelou que a prevalência de diabéticos com risco aumentado de ulceração a nível dos Cuidados de Saúde Primários é significativa, realçando a necessidade de uma detecção precoce das alterações no pé e investimento na educação dos diabéticos sobre os cuidados a ter com os pés.

Palavras-Chave: Diabetes Mellitus; Pé Diabético; Cuidados Primários de Saúde.

\section{INTRODUÇÃO}

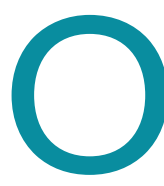

pé diabético é umas das complicações mais graves da Diabetes Mellitus. ${ }^{1,2}$ As complicações nos pés como ulceração, infecção e gangrena condicionam elevada morbilidade e são as principais causas de hospitalização do doente diabético, correspondendo a cerca de $20 \%$ das admissões hospitalares nestes doentes. São igualmente responsáveis por 40-60\% das amputações não traumáticas do membro inferior no mundo industrializado. ${ }^{2,3,4}$ Estima-se que o risco de amputação do membro inferior é 15 a 46 vezes mais elevado em doen-

\footnotetext{
*Assistente de Medicina Geral e Familiar, Centro de Saúde da Senhora da Hora (Unidade Local de Saúde de Matosinhos)

**Interna de Medicina Geral e Familiar, Centro de Saúde de Leça da Palmeira (Unidade Local de Saúde de Matosinhos)

***Assistente de Medicina Geral e Familiar, Unidade de Saúde Familiar Infesta (Unidade Local de Saúde de Matosinhos)
}

tes com Diabetes Mellitus do que em pessoas que não padecem desta doença, sendo a incidência de amputação considerada como o principal marcador de qualidade de cuidados do doente diabético. ${ }^{4,5}$ A incidência anual ajustada para a idade de amputação não traumática dos doentes diabéticos varia de 2,1 a 13,7 por 1000 indivíduos e a mortalidade pós-amputação é de 13 a $40 \%$ no primeiro ano, 35 a $65 \%$ aos 3 anos e 39 a $80 \%$ aos 5 anos após a amputação. ${ }^{6}$ Estima-se que o custo de tratamento de um pé diabético com úlcera corresponda a cerca de $\$ 18000$ (dólares americanos de 2000) nos casos sem amputação, ascendendo a $\$ 34000$ nos doentes que necessitam de amputação. ${ }^{6}$

A lesão mais característica do pé diabético é a úlcera, que é um dos principais factores de risco e precede em 85\% dos casos a amputação do membro inferior. ${ }^{1,3,4,6}$ Têm sido identificados vários factores de risco para o desenvolvimento de úlceras em doentes com Diabetes Melli- 
tus. Uma história prévia de ulceração ou amputação do membro inferior é o principal factor associado à (re)ocorrência de ulceração, estimando-se que $70 \%$ das úlceras podem recorrer num período de 5 anos após o tratamento inicial. A neuropatia periférica desempenha um papel central na etiopatogenia das úlceras em diabéticos, aumentando o risco de ulceração em 8 a 18 vezes. Outros factores causais de ulceração são a presença de deformidades do pé (frequentemente relacionadas com a neuropatia periférica) e a limitação da mobilidade articular, condicionando alterações da biomecânica podálica e pressão plantar excessiva. A doença arterial periférica tem também um papel importante no pé dos diabéticos, sendo o factor prognóstico mais importante numa úlcera do pé. Têm sido também descritos outros factores de risco em vários estudos, tais como: idade avançada, maior duração da diabetes, retinopatia, nefropatia, mau controlo glicémico, insulinoterapia, tabagismo, alcoolismo, tipo de calçado e condições socioeconómicas deficientes. No entanto, a evidência é insuficiente para a utilização destes factores como preditivos da ocorrência de ulceração em diabéticos. ${ }^{3,4,7-13}$

Em 1999, o International Working Group on the Diabetic Foot (IWGDF) desenvolveu um sistema de classificação de pé diabético onde são identificados 4 grupos de risco. $O$ grupo 0 consiste em pessoas sem neuropatia periférica significativa confirmada apenas pela sua capacidade em sentir o monofilamento de Semmes-Weinstein 5.07 de $10 \mathrm{~g}$ (doentes de baixo risco). O grupo 1 inclui doentes com neuropatia isolada; o grupo 2 engloba doentes neuropáticos com deformidades no pé ou doença arterial periférica; e o grupo 3 abrange aqueles com história prévia de ulceração ou amputação de membro inferior. Os doentes pertencentes aos grupos 1, 2 e 3 têm risco aumentado de ulceração. ${ }^{1}$ A eficácia desta classificação em predizer complicações do pé em doentes diabéticos foi demonstrada num estudo prospectivo $^{11}$, sendo que o risco de ulceração aos 3 anos, de $5,1 \%$ no grupo 0 , aumentou para $14,3 \%, 18,8 \%$ e $55,8 \%$ nos grupos 1,2 e 3 , respectivamente. ${ }^{10}$

Em Portugal estima-se que cerca de 15 por cento da população diabética tenha condições favoráveis ao aparecimento de lesões nos pés e que possam ocorrer anualmente cerca de 500 amputações não traumáticas dos membros inferiores, resultando num esforço acrescido do membro remanescente, que iniciará proble- mas em ano e meio. ${ }^{2}$ Existem poucos estudos publicados sobre este problema na população portuguesa, especialmente num contexto de cuidados primários. Um estudo realizado na região norte do país identificou uma prevalência de neuropatia diabética de $32,2 \% .^{14}$ No entanto, nenhum estudo foi realizado em Portugal com vista a avaliar a prevalência de doentes diabéticos com pé em risco aumentado de ulceração usando a classificação da IWGDF. Este é um problema que, dada a sua morbimortalidade e suposta prevalência, numa doença cuja história natural é longa, necessita da atenção da comunidade médica uma vez que existem estratégias comprovadas de prevenção, aconselhamento e tratamento destes doentes.

Com este estudo pretendeu-se:

- Determinar a prevalência de pé em risco aumentado de ulceração em doentes diabéticos tipo 2 no concelho de Matosinhos, utilizando a classificação do IWGDF;

- Determinar a associação entre o risco aumentado de ulceração e outras variáveis da população em estudo como idade, sexo, escolaridade, situação profissional, obesidade, período desde diagnóstico e terapêutica da diabetes e controlo glicémico.

\section{MÉTODOS}

Realizou-se um estudo observacional analítico transversal, com recolha de dados entre Junho e Setembro de 2007, nos Centros de Saúde de Leça da Palmeira, Senhora da Hora e S. Mamede de Infesta, do concelho de Matosinhos, Portugal.

A população em estudo envolveu todos os doentes com Diabetes mellitus (DM) tipo 2 inscritos nos Centros de Saúde atrás referidos que cumpriam os seguintes critérios de inclusão:

- Doentes inscritos nos Centros de Saúde onde o estudo decorreu;

- Doentes portadores de Guia do Diabético;

- Doentes com residência permanente em Portugal. Foram excluídos do estudo os doentes que apresentavam as seguintes características:

- Acamados ou com outras incapacidades que impediram a sua deslocação até ao Centro de Saúde;

- Doentes com demência conhecida;

- Doentes com amputação bilateral do membro inferior; 
- Doentes com úlcera activa.

Considerando um nível de precisão de $5 \%$ e intervalo de confiança de $95 \%$, com uma prevalência esperada de pé com risco aumentado de ulceração em Diabéticos tipo 2 de $15 \%$, de acordo com a Circular Normativa N. ${ }^{\circ}$ 8/DGCG de 24/04/2001 da Direcção Geral de Saúde referente ao Pé Diabético, ${ }^{2}$ calculou-se ser necessária uma amostra de 237 pessoas. A técnica de amostragem foi aleatória simples com reposição, tendo como base a lista de diabéticos inscritos nos Centros de Saúde e portadores de Guia do Diabético conforme registo no sistema informático SINUS (Sistema de Informatização nas Unidades de Saúde). A partir de uma lista ordenada alfabeticamente, foram aleatorizados 237 utentes diabéticos com o auxílio do programa informático Random.org disponível on-line (URL disponível em http://www.random.org/).

\section{Recolha de informação}

Os doentes foram contactados via telefone e foi explicada a finalidade do estudo, tendo sido convidados para uma entrevista individual com observação directa dos pés realizada pelo investigador no Centro de Saúde em que o doente se encontrava inscrito. As chamadas foram feitas ao longo de um dia útil de trabalho. Quando um doente não atendia a chamada, esta era repetida no final do dia e no dia seguinte. Se o doente não voltava a atender, ou recusava participar, era realizada reposição da amostra com o doente imediatamente a seguir na lista total. Na entrevista foi novamente explicada ao doente a finalidade do estudo, entregue um documento informativo do mesmo e obtido o consentimento informado para a sua participação. Foram registados numa folha especificamente desenvolvida para tal os dados relativos à idade, género, nível de escolaridade, situação profissional, período desde diagnóstico e terapêutica da Diabetes e antecedentes de úlcera e amputação com base em informação dada pelo doente. Através de consulta do processo clínico de cada doente foi obtido e registado o último valor de hemoglobina glicosilada (HbAlc) no último ano. Por observação directa foram medidos o peso e altura e verificada a existência ou não de úlcera activa, bem como a presença de deformidades, presença de pulso arterial periférico e presença de neuropatia periférica.

\section{Definição das variáveis}

Idade - definida através de 3 categorias (idade inferior a 40 anos; idade compreendida entre os 40 e 60 anos; idade superior a 60 anos).

Género - definido como masculino ou feminino.

Nível de escolaridade - definido através de 7 categorias (analfabeto; sabe ler e escrever; ensino primário completo; ensino básico completo; ensino secundário completo e ensino superior completo).

Situação profissional - definida através de 5 categorias (desempregado; trabalhador no activo; reformado; dona de casa e outra situação).

Índice de Massa Corporal - definido em 4 categorias (peso normal se inferior a 25; excesso de peso se compreendido entre 25 e 29,9; obesidade se compreendido entre 30 e 34,9 e obesidade mórbida se superior a 35$).^{15}$

Controlo glicémico - definido através do último registo de HbAlc avaliada até 1 ano antes do início do estudo, constante no processo clínico do doente. Classificou-se como bom controlo se HbAlc $<$ ou $=7 \%$ ou mau controlo se HbAlc $>7 \%{ }^{16}$; sem registo se não houvesse registo do valor $\mathrm{HbA}_{\mathrm{lc}}$ relativo ao ano prévio ao estudo.

Período desde diagnóstico da DM - definidas 5 categorias (inferior a 5 anos; compreendido entre 5 e 10 anos; compreendido entre 10 e 15 anos; compreendido entre 15 e 20 anos e superior a 20 anos).

Tratamento actual da diabetes - definida através de 2 categorias (insulinotratados e não insulinotratados).

Doentes com pé em risco aumentado de ulceração - definido como doentes incluídos nos grupos 1, 2 ou 3 do Sistema de Classificação do International Working Group on the Diabetic Foot de 1999 ${ }^{1,10,11}$ que apresenta 4 grupos de risco crescente:

Grupo 0: sem neuropatia periférica significativa;

Grupo 1: com neuropatia periférica isolada;

Grupo 2: com neuropatia periférica e deformidades do pé ou doença vascular periférica;

Grupo 3: doentes com história prévia de úlcera ou amputação.

\section{Instrumentos de medida}

A neuropatia periférica foi pesquisada através da utilização do monofilamento de Semmes-Weinstein 5.07 de 10 gramas, de acordo com a técnica proposta pelo International Working Group on Diabetic Foot. ${ }^{1}$ Em pri- 
meiro lugar, o monofilamento foi aplicado nas mãos do doente (ou cotovelo ou testa) de modo a que o doente percebesse o que devia esperar sentir. $O$ doente não conseguia ver se o examinador aplicava o monofilamento nem em que local. Os locais de pesquisa de sensibilidade incluíram o ápice do hálux e a área correspondente à primeira e quinta cabeças metatársicas, na planta de ambos os pés. Aplicou-se o monofilamento perpendicularmente à superfície da pele, com uma força suficiente para fazer o filamento curvar ou entortar. O tempo total de duração da aproximação, contacto com a pele e retirada do monofilamento foi cerca de dois segundos. Pressionado o monofilamento contra a pele foi perguntado ao doente se sentia a pressão aplicada (sim/não) e, a seguir, onde sentia a mesma (pé esquerdo/direito). Repetiu-se esta aplicação duas vezes no mesmo local, alternando com, pelo menos, uma aplicação «falsa» em que não era aplicado qualquer monofilamento (total de três perguntas por local). Foi considerado pé neuropático quando em pelo menos um local de aplicação do monofilamento se obtiveram duas respostas erradas. O monofilamento foi utilizado em até 10 doentes por sessão e só foi novamente utilizado 24 horas depois de forma a manter a sua força tensional. Um monofilamento de 10 gramas novo e idêntico foi fornecido a cada investigador com o intuito de unificar e garantir reprodutibilidade na pesquisa de neuropatia periférica.

A presença de vasculopatia periférica foi avaliada pela palpação dos pulsos arteriais das artérias pediosa e tibial posterior (artéria pediosa: pesquisa de pulso arterial na região medial do dorso do pé, com o pé ligeiramente dorsiflectido; artéria tibial posterior: pesquisa do pulso arterial posteriormente e ligeiramente inferior ao maléolo medial do tornozelo). ${ }^{17}$ Foi considerado pé com vasculopatia aquele em que havia ausência dos dois pulsos pesquisados.

No exame objectivo do pé, foram consideradas como alterações as proeminências ósseas, as calosidades, os dedos em garra/martelo e a pele seca, fissurada e com presença de tinha dos pés ou onicomicose. ${ }^{6,18}$

O peso e a estatura, para cálculo do IMC, foram medidos com os utentes descalços, sem casaco ou qualquer acessório nos bolsos. A avaliação do peso foi feita com uma balança de craveira calibrada, com o utente no centro da balança de forma a distribuir o peso uni- formemente pelos dois pés. A avaliação da estatura foi feita com um estadiómetro fixo, em pé, com os calcanhares unidos e a cabeça posicionada no plano horizontal de Frankfurt. O IMC foi calculado dividindo o peso (quilogramas) pelo quadrado da estatura (metros)..$^{15}$

Os quatro investigadores tiveram uma sessão de treino das técnicas entre eles para unificação dos procedimentos.

\section{Tratamento dos dados}

Os dados recolhidos foram codificados e posteriormente registados numa base de dados informática usando o software Excel for Windows ${ }^{\circledast}$. Utilizou-se o programa SPSS versão $15.0^{\circledR}$ para o tratamento estatístico dos mesmos. Foram determinados resultados referentes à estatística descritiva e à estatística inferencial. A comparação de proporções foi efectuada utilizando a teste Qui-quadrado. O nível de significância (p) adoptado para aceitação de diferenças estatisticamente significativas foi 0,05 .

\section{RESULTADOS}

As principais características da amostra encontram-se descritas no Quadro I. Foram avaliados 237 doentes diabéticos, sendo que $46 \%$ eram do sexo masculino. A média de idades encontrada foi de 63 anos, com um desvio padrão de 11 anos. Uma grande maioria dos indivíduos estudados $(46,4 \%)$ tinha um valor de índice de massa corporal entre 25 e $29,9 \mathrm{~kg} / \mathrm{m}^{2}$. Em relação ao nível de escolaridade, há a salientar que 59,9\% dos doentes tinha o ensino primário completo. Do total de diabéticos, 59,5\% estavam reformados. A Diabetes Mellitus havia sido diagnosticada há menos de 10 anos em cerca de $60,7 \%$ dos doentes e $46,8 \%$ apresentavam uma HbAlc superior a $7 \%$. Apenas $8 \%$ se encontravam em tratamento com insulina.

A prevalência encontrada de pé em risco aumentado de ulceração foi de 18,1\% (Gráfico 1). Quando analisada a prevalência por grupo de risco, encontram-se os seguintes resultados: grupo 1- 3,8\%; grupo $2-11,0 \%$ e grupo 3- 3,3\%.

Em relação às alterações encontradas nos pés dos doentes estudados, verificou-se que $59,5 \%$ apresentava deformidades, $16,5 \%$ tinha critérios de neuropatia periférica, $5,9 \%$ não tinha pulsos periféricos palpáveis 


\begin{tabular}{|c|c|}
\hline \multicolumn{2}{|c|}{ QUADRO I. Caracterização da amostra estudada } \\
\hline & $\mathrm{n}(\%)^{*}$ \\
\hline \multicolumn{2}{|l|}{ Idade (anos) } \\
\hline$<40$ & $6(2,5)$ \\
\hline $40-60$ & $80(33,8)$ \\
\hline $60-75$ & $119(50,2)$ \\
\hline$>75$ & $32(13,5)$ \\
\hline \multicolumn{2}{|l|}{ Sexo } \\
\hline Masculino & $109(46)$ \\
\hline Feminino & $128(54)$ \\
\hline \multicolumn{2}{|l|}{ Nível de Escolaridade } \\
\hline Analfabeto & $17(7,2)$ \\
\hline Sabe ler e escrever & $32(13,5)$ \\
\hline Ensino Primário Completo & $142(59,9)$ \\
\hline Ensino Básico Completo & $23(9,7)$ \\
\hline Ensino Secundário Completo & $16(6,8)$ \\
\hline Ensino Superior Completo & $7(3)$ \\
\hline \multicolumn{2}{|l|}{ Situação Profissional } \\
\hline Desempregado & $54(22,8)$ \\
\hline Trabalhador no Activo & $20(8,4)$ \\
\hline Reformado & $141(59,5)$ \\
\hline Dona de Casa & $16(6,8)$ \\
\hline Outros & $6(2,5)$ \\
\hline \multicolumn{2}{|l|}{ IMC $\left(\mathrm{kg} / \mathrm{m}^{2}\right)$} \\
\hline$<25,0$ & $23(9,7)$ \\
\hline $25,0-29,9$ & $110(46,4)$ \\
\hline $30-34,9$ & $72(30,4)$ \\
\hline$\geq 35$ & $23(9,7)$ \\
\hline \multicolumn{2}{|l|}{ Controlo Glicémico (HbA1c) } \\
\hline$<7 \%$ & $96(46,8)$ \\
\hline$\geq 7 \%$ & $109(46,0)$ \\
\hline \multicolumn{2}{|c|}{ Período desde diagnóstico da Diabetes (anos) } \\
\hline $0-4$ & $83(35)$ \\
\hline $5-9$ & $61(25,7)$ \\
\hline $10-14$ & $37(15,6)$ \\
\hline $15-19 \S$ & $25(10,5)$ \\
\hline$\geq 20$ & $31(13,1)$ \\
\hline \multicolumn{2}{|l|}{ Tratamento da Diabetes } \\
\hline Insulinotratados & $19(8)$ \\
\hline Não insulinotratados & $218(92)$ \\
\hline
\end{tabular}

*O total pode ser diferente de $100 \%$ por indisponibilidade de informação.

e 3,4\% contavam uma história passada de úlcera ou de amputação, conforme descrito no Gráfico 2.

Quando comparados os pacientes sem risco aumentado de ulceração com aqueles que se encontram

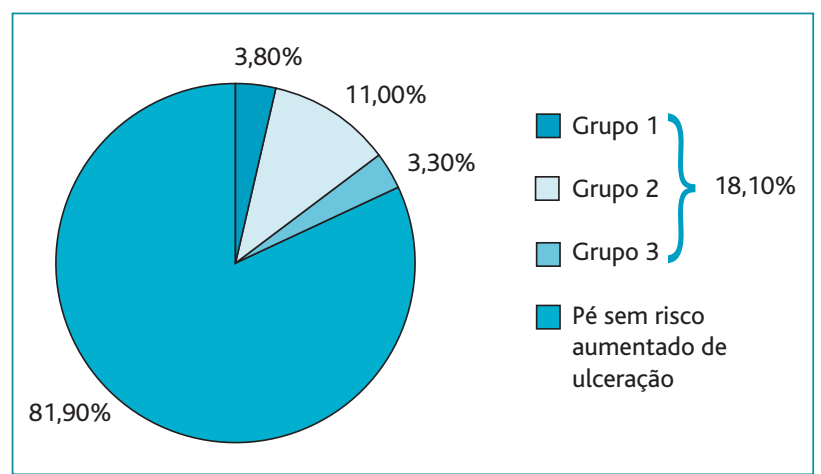

Gráfico 1. Prevalência de pé sem risco e dos diferentes grupos de pé em risco de ulceração.

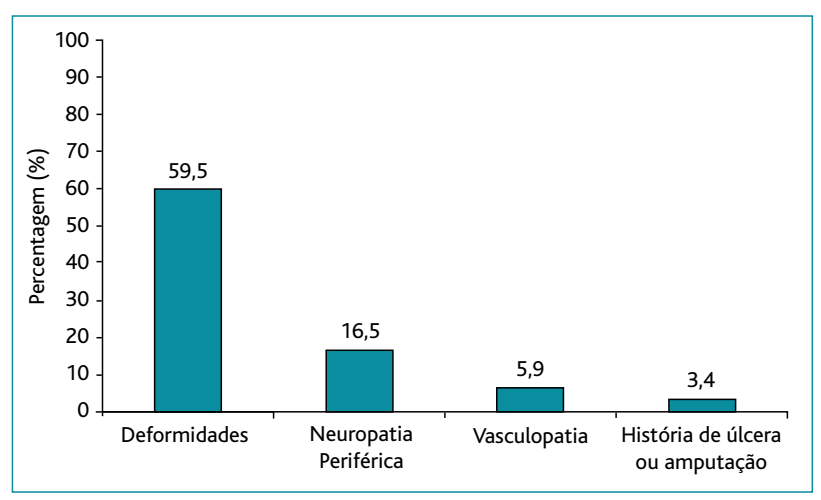

Gráfico 2. Prevalência das alterações encontradas nos pés em risco.

na categoria de risco aumentado, encontramos uma associação positiva para a situação profissional, sendo que há mais reformados com pé em risco aumentado de ulceração $(\mathrm{p}<0,05)$. Admitindo que a idade poderia ser um factor de confundimento, foi realizada uma regressão logística comparando a variável dependente com a idade e a situação profissional, verificando-se que a relação entre pé em risco aumentado de ulceração e a situação profissional é independente da idade $(\mathrm{p}=0,007)$. Comparando o grupo de diabéticos insulinotratados com aqueles que não utilizam insulina, verificou-se também uma diferença mas sem significado estatístico. Não foram encontradas associações estatisticamente significativas com as restantes variáveis. As associações entre as diversas variáveis estudadas encontram-se descritas no Quadro II. 


\begin{tabular}{|c|c|c|c|}
\hline \multicolumn{4}{|c|}{$\begin{array}{l}\text { QUADRO II. Comparação entre as variáveis estudadas e a variável pé em risco } \\
\text { aumentado de ulceração (Teste Estatístico: Qui-Quadrado) }\end{array}$} \\
\hline & $\begin{array}{c}\text { Com risco } \\
\text { aumentado } \\
\text { de ulceração } \\
\text { n (\%) }\end{array}$ & $\begin{array}{c}\text { Sem risco } \\
\text { aumentado } \\
\text { de ulceração } \\
\text { n (\%) }\end{array}$ & Valor de $p$ \\
\hline \multicolumn{4}{|l|}{ Idade (anos) } \\
\hline$<40$ & $0(0,0)$ & $6(100,0)$ & \multirow{4}{*}{0,294} \\
\hline $40-60$ & $11(13,7)$ & $69(86,3)$ & \\
\hline $60-75$ & $24(20,2)$ & $95(79,8)$ & \\
\hline$>75$ & $8(25)$ & $24(75)$ & \\
\hline \multicolumn{4}{|l|}{ Sexo } \\
\hline Masculino & $17(15,6)$ & $92(84,4)$ & \multirow[t]{2}{*}{0,348} \\
\hline Feminino & $26(20,3$ & $102(79,7)$ & \\
\hline \multicolumn{4}{|l|}{ Nível de Escolaridade } \\
\hline Analfabeto & $3(17,6)$ & $14(82,4)$ & \multirow{6}{*}{0,136} \\
\hline Sabe ler e escrever & $11(34,4)$ & $21(65,6)$ & \\
\hline Ensino Primário Completo & $22(15,5)$ & $120(84,5)$ & \\
\hline Ensino Básico Completo & $4(17,4)$ & $19(82,6)$ & \\
\hline Ensino Secundário Completo & $1(6,3)$ & $15(93,8)$ & \\
\hline Ensino Superior Completo & $2(28,6)$ & $5(71,4)$ & \\
\hline \multicolumn{4}{|l|}{ Situação Profissional } \\
\hline Desempregado & $4(7,4)$ & $50(92,6)$ & \multirow{5}{*}{$<0,05$} \\
\hline Trabalhador no Activo & $1(5,0)$ & $19(95,0)$ & \\
\hline Reformado & $34(24,1)$ & $107(75,9)$ & \\
\hline Dona de Casa & $4(25,0)$ & $12(75,0)$ & \\
\hline Outros & $0(0)$ & $6(100,0)$ & \\
\hline \multicolumn{4}{|l|}{$\mathrm{IMC}\left(\mathrm{kg} / \mathrm{m}^{2}\right)$} \\
\hline$<25,0$ & $5(21,7)$ & $18(78,3)$ & \multirow{4}{*}{0,252} \\
\hline $25,0-29,9$ & $21(19,1)$ & $89(80,9)$ & \\
\hline $30-34,9$ & $9(12,5)$ & $63(87,5)$ & \\
\hline$\geq 35$ & $7(30,4)$ & $16(69,6)$ & \\
\hline \multicolumn{4}{|l|}{ Controlo Glicémico (HbA1c) } \\
\hline$<7 \%$ & $15(15,6)$ & $81(84,4)$ & \multirow[t]{2}{*}{0,314} \\
\hline$\geq 7 \%$ & $23(21,1)$ & $86(78,9)$ & \\
\hline \multicolumn{4}{|c|}{ Período desde diagnóstico da Diabetes (anos) } \\
\hline $0-4$ & $13(15,7)$ & $70(84,3)$ & \multirow{5}{*}{0,198} \\
\hline $5-9$ & $10(16,4)$ & $51(83,6)$ & \\
\hline $10-14$ & $6(16,2)$ & $31(83,8)$ & \\
\hline $15-19$ & $9(36,0)$ & $16(64,0)$ & \\
\hline$\geq 20$ & $5(16,1)$ & $26(83,9)$ & \\
\hline \multicolumn{4}{|l|}{ Tratamento da Diabetes } \\
\hline Insulinotratados & $7(36,8)$ & $12(63,2)$ & \multirow[t]{2}{*}{0,173} \\
\hline Não insulinotratados & $36(16,5)$ & $182(83,5)$ & \\
\hline
\end{tabular}

de $18,1 \%$ dos doentes diabéticos tem um risco aumentado de vir a desenvolver úlcera no pé, verificando-se uma relação estatisticamente significativa com o facto de estarem reformados. Não se encontrou associação entre o risco de ulceração e outros factores, nomeadamente o controlo glicémico, a obesidade, período desde diagnóstico da diabetes e tipo de terapêutica.

O presente estudo é o primeiro publicado em Portugal com vista a avaliar a prevalência de doentes diabéticos com pé em risco aumentado de ulceração utilizando a classificação proposta pelo IWGDF. Como é realizado em contexto de Cuidados de Saúde Primários, evita a sobre-representação de doentes com maior número e gravidade de complicações da diabetes observado em populações clínicas de base hospitalar.

O sistema de classificação adoptado encontra-se validado, permitindo, através da utilização de métodos simples e facilmente disponíveis na prática clínica, a estratificação de doentes em grupos de risco preditivos de complicações. ${ }^{1,10}$

Utilizou-se o monofilamento de Semmes-Weinstein 5.07 de 10 gramas para a pesquisa de neuropatia periférica que é um método recomendado pela Organização

\section{DISCUSSÃO}

Após a análise destes resultados constata-se que cerca
Mundial de Saúde para a identificação de diabéticos em risco de ulceração. ${ }^{19}$ Tem baixos custos, é prático, re- 
produtível, validado e mais preditivo de risco de ulceração que a pesquisa da sensibilidade vibratória com o diapasão de $128 \mathrm{~Hz} .^{11,20,21}$ Seguiu-se a técnica proposta pelo IWGDF, tendo-se pesquisado a sensibilidade em três localizações em cada pé, que tem a vantagem de ser prática e rápida mantendo uma sensibilidade $(80 \%)$ e especificidade ( $86 \%$ ) adequadas. ${ }^{1,6,9,11}$

A escala adoptada não considera os doentes com doença vascular periférica isolada como um grupo de risco uma vez que tem sido debatido o seu papel na etiologia das úlceras em diabéticos, sugerindo-se que a doença vascular possa ser um factor de risco mais importante para a cicatrização mais prolongada da úlcera e subsequente amputação do que para o seu aparecimento. ${ }^{6,10,11}$ A pesquisa da vasculopatia foi efectuada através da palpação de pulsos periféricos que é uma técnica facilmente exequível e aceite para a pesquisa de doença vascular periférica ${ }^{6,11}$ Não foram utilizados testes vasculares não invasivos uma vez que estes não estão tão facilmente disponíveis na prática clínica.

As principais limitações do estudo incluem a selecção dos doentes, a variabilidade inter-observador e a colheita de dados relativos a algumas variáveis.

$O$ facto de a amostra ter sido obtida com base na lista de diabéticos inscritos nos Centros de Saúde conforme registo no sistema informático SINUS origina um viés de selecção na medida em que nem todos os doentes diabéticos se encontram registados no programa. No entanto, este consiste na fonte de dados mais facilmente disponível e completa para identificação dos doentes.

Por outro lado, como a colheita dos dados foi efectuada por quatro investigadores, estes podem estar sujeitos a um viés de medição, que foi minimizado com a uniformização das técnicas e material a ser utilizado.

Relativamente às variáveis em estudo, a obtenção dos valores de $\mathrm{HbAlc}$ foi feita com base nos processos clínicos, condicionando um viés de registo, tendo-se verificado ausência de dados em $13,5 \%$ dos casos. Na colheita da informação relativa ao período desde diagnóstico da diabetes, poderá estar subjacente um viés de memória, tendo em conta que foi obtida através de informação oral do doente.

A prevalência de pé em risco aumentado de ulceração encontrada neste estudo foi de $18,1 \%$, valor ligeiramente superior ao estimado pela DGS (15\%). Este va- lor é também ligeiramente superior ao encontrado num estudo francês que, embora de base hospitalar, é metodologicamente semelhante a este trabalho. Utilizando também a classificação em grupos de risco da IWGDF, Malgrange et al encontrou uma prevalência de pé em risco aumentado de $17,5 \% .^{11}$

Em relação às alterações encontradas nos pés dos diabéticos, verificou-se que as deformidades foram as mais prevalentes (59,5\%). Este resultado não pode ser comparado com o de outros estudos uma vez que não há homogeneidade na definição e avaliação desta variável entre os vários estudos.

A prevalência de neuropatia periférica varia de acordo com diferentes estudos, reflectindo diferenças em termos de população estudada (hospitalar versus comunitária) bem como de métodos de pesquisa utilizados. Assim, a prevalência de neuropatia periférica encontrada $(16,5 \%)$ foi inferior à de outros trabalhos realizados em meio hospitalar ${ }^{11,22}$ e que incluíam doentes com úlcera activa, ou seja, doentes com maior gravidade de complicações, o que justifica a sobre-representação de doentes com neuropatia neste meio.

Num outro estudo realizado no norte de Portugal, ${ }^{14}$ numa amostra de 93 doentes, no âmbito de Cuidados Primários, foi encontrada uma prevalência muito mais elevada de neuropatia, $32,2 \%$. Este facto poderá dever-se a diferenças em termos de critérios de diagnóstico da neuropatia. Esse estudo avaliou a presença de neuropatia pela pesquisa de sintomas neuropáticos e de alterações da sensibilidade vibratória, propioceptiva, dolorosa e térmica. No presente estudo apenas foi utilizado o monofilamento por ser mais preditivo de risco de ulceração do que a pesquisa das outras sensibilidades e mais objectivo do que os sintomas neuropáticos.

A presença de vasculopatia (5,9\%), pesquisada através da palpação dos pulsos periféricos, foi também menos prevalente que no estudo de Malgrange et $\mathrm{al}^{11}$ (17\%), o qual foi realizado numa população de base hospitalar. Outros estudos que utilizaram outros métodos de pesquisa, como índice tornozelo-braço, apresentaram prevalências de vasculopatia mais elevadas. ${ }^{23}$ No entanto, optou-se por determinar a prevalência desta complicação através de uma técnica simples, de fácil execução na prática clínica.

A presença de história de úlcera prévia ou amputação $(3,4 \%)$ foi também menos prevalente neste estudo que noutros de âmbito hospitalar., ${ }^{9,1}$ 
Verificou-se uma associação positiva entre risco aumentado de ulceração e situação profissional, sendo maior o risco nos reformados. Após análise multivariada por regressão logística esta relação foi independente da idade. Esta associação poder-se-á explicar pelo facto dos reformados apresentarem uma vida mais sedentária (possível variável de confundimento), o que não foi avaliado neste estudo. Também não existem outros estudos que tenham avaliado a relação entre sedentarismo e maior risco de ulceração.

Vários estudos encontraram relação entre idade e neuropatia ou risco aumentado de ulceração, embora não tenham realizado análise multivariada, pelo que a idade pode ser uma variável de confundimento da associação entre risco de ulceração e período desde diagnóstico da diabetes, também verificada nesses estudos. ${ }^{11,14,22}$ Neste estudo, apesar de se ter verificado uma tendência para um aumento do risco de ulceração com a idade, esta não foi estatisticamente significativa.

Tal como noutros estudos, não se encontrou associação entre o risco aumentado de ulceração e o género. ${ }^{11,14,20,22,24}$

Não se encontrou relação entre o valor de IMC e o risco de ulceração, o que também não se verificou noutros estudos. ${ }^{11,14}$ No estudo prospectivo de Boyko et al foi encontrada relação entre risco de ulceração e o IMC. ${ }^{9}$

Verificou-se que a prevalência de risco aumentado de ulceração foi maior no grupo de doentes com HbAlc $\geq 7 \%$, mas essa diferença não foi significativa, o que também ocorreu noutros estudos. ${ }^{7,8,9,14}$ O UKPDS mostrou não haver impacto consistente do controlo glicémico no desenvolvimento de neuropatia na DM tipo $2 .{ }^{25}$ No entanto, alguns estudos obtiveram resultados diferentes, demonstrando associação positiva entre o mau controlo glicémico e o desenvolvimento de neuropatia e microangiopatia. ${ }^{22}$

Quanto ao período desde diagnóstico da diabetes também não se encontrou associação com o risco aumentado de ulceração. São muito controversos os resultados dos estudos que analisaram esta relação: há estudos que encontraram associação $0^{7,11,14,20,22}$ e outros que não, ${ }^{9}$ esta diferença de resultados pode ser explicada pela dificuldade em estabelecer o tempo exacto de duração da diabetes.

Em relação ao tratamento da diabetes, não se verificou diferença estatisticamente significativa entre o ris- co de ulceração nos doentes insulinotratados e nos que não utilizam insulina. Há estudos que demonstraram essa associação, mas todos realizados em populações de base hospitalar, tendo um maior número de doentes insulinotratados que poderá explicar essa diferença de resultados. ${ }^{8,9}$

Outros trabalhos avaliaram a relação entre o risco de neuropatia/ulceração e outros factores que não foram avaliados neste estudo, tendo sido encontrada associação com nefropatia, ${ }^{11}$ retinopatia, ${ }^{9,11,20} \mathrm{e}$ doença coronária ${ }^{8,14}$. Apesar de ter sido encontrada relação entre hábitos tabágicos e risco de ulceração num estudo ${ }^{7}$, outros têm reportado resultados opostos ${ }^{8,9,14,22}$. O mesmo se verifica em relação à hipertensão arterial. ${ }^{14,26}$

Este estudo revelou que a prevalência de diabéticos com risco aumentado de ulceração a nível dos Cuidados de Saúde Primários no concelho de Matosinhos é significativa. Foi também possível conhecer a prevalência de alterações nos pés dos diabéticos como deformidades (presentes em mais de metade dos diabéticos), neuropatia, ausência de pulsos ou história de úlcera prévia ou amputação.

Os resultados deste estudo enfatizam a necessidade de uma detecção precoce das alterações no pé, de forma a diminuir o risco de ulceração e amputação. Demonstrou-se como, através da colheita de história clínica, realização de exame objectivo e aplicação de técnicas simples como monofilamento e palpação dos pulsos periféricos, é possível determinar o grau de risco de ulceração, o que determina o tipo e frequência das intervenções necessárias. É necessário que os clínicos invistam numa melhoria contínua de cuidados que passa pela educação dos diabéticos sobre os cuidados a ter com os pés bem como pela necessidade da observação periódica dos pés de todos os diabéticos de forma a avaliar o risco de ulceração.

Neste estudo a situação de reformado parece aumentar o risco de ulceração nos doentes diabéticos, no entanto são necessários estudos prospectivos para confirmar esta relação. Seria também interessante, em futuros estudos, investigar os factores que poderão contribuir para um maior risco de ulceração nos reformados, como por exemplo o sedentarismo.

REFERÊNCIAS BIBLIOGRÁFICAS

1. Apelqvist J, Bakker K, van Houtum WH, Nabuurs-Franssen MH, Schaper 
NC. International consensus and practical guidelines on the management and the prevention of the diabetic foot. Diabetes Metab Res Rev 2000 Sep-Oct; 16 (Suppl 1): S84-S92.

2. Divisão de Doenças Genéticas, Crónicas e Geriátricas. Pé diabético Programa de controlo da Diabetes Mellitus. DGS. Circular Normativa No 8/DGCG de 24/04/2001.

3. Frykber RG. Diabetic foot ulcers: pathogenesis and management. Am Fam Physician 2002 Nov 1; 66(9): 1655-62.

4. Armstrong DG, Lavery LA. Diabetic foot ulcers: prevention, diagnosis and classification. Am Fam Physician 1998 Mar 15; 57 (6): 1325-32, 1337-8.

5. Jeffcoate WJ, Van Houtum WH. Amputation as a marker of the quality of foot care in diabetes. Diabetologia 2004 Dec; 47 (12): 2051-8.

6. Singh N, Armstong DG, Lipsky BA. Preventing foot ulcers in patients with diabetes. JAMA 2005 Jan 12; 293 (2):217-28.

7. de Sonnaville JJ, Colly LP, Wijkel D, Heine RJ. The prevalence and determinants of foot ulceration in type II diabetic patients in a primary health care setting. Diabetes Res Clin Pract 1997 Mar; 35(2-3):149-56.

8. Adler Al, Boyko EJ, Ahroni JH, Smith DG. Lower-extremity amputation in diabetes: the independent effects of peripheral vascular disease, sensory neuropathy, and foot ulcers. Diabetes Care 1999 Jul; 22 (7):102935.

9. Boyko EJ, Ahroni JH, Stensel V, Forsberg RC, Davignon DR, Smith DG. A prospective study of risk factors for diabetic foot ulcer. Diabetes Care 1999 Jul; 22 (7):1036-42.

10. Peters EJ, Lavery LA; International Working Group on the Diabetic Foot. Effectiveness of the diabetic foot risk classification system of the International Working Group on the Diabetic Foot. Diabetes Care 2001 Aug; 24 (8): 1442-7.

11. Malgrange D, Richard JL, Leymarie F; French Working Group on the Diabetic Foot. Screening diabetic patients at risk for foot ulceration: a multi-centre hospital-based study in France. Diabetes Metab 2003 Jun; 29 (3): 261-8.

12. Leymarie F, Richard JL, Malgrange D. Factors associated with diabetic patients at high risk for foot ulceration. Diabetes Metab 2005 Dec; 31 (6): 603-5.

13. Revilla GP, Sá AB, Carlos JS. O pé dos diabéticos. Rev Port Clin Geral 2007 Set-Out; 23 (5): 615-26.

14. Barbosa AP, Medina JL, Ramos EP, Barros HP. Prevalence and risk factors of clinical diabetic polyneuropathy in a Portuguese primary health care population. Diabetes Metab 2001 Sep; 27 (4 Pt 1): 496-502.

15. Kuczmarsk RJ, Ogden CL, Grummer-Strawn LM, Flegal KM, Guo SS, Wei R, et al. CDC growth charts: United States. Adv Data 2000 Jun 8; (314): 1-27.

16. Divisão de Doenças Genéticas, Crónicas e Geriátricas. Actualização dos critérios de classificação e diagnóstico da Diabetes Mellitus. DGS. Circular Normativa No 9/DGCG de 04/07/2002.
17. Seidel HE, Ball JW, Dains JE, Benedict GW, editors. Mosby's Guide to Physical Examination. 4th ed. Local: Dt. Louis, MO: Mosby; 1999. p. 445.

18. Boulton AJ. Lowering the risk of neuropathy, foot ulcers and amputations. Diabet Med 1998; 15 Suppl 4: S57-S59.

19. Lee S, Kim H, Choi S, Park Y, Kim Y, Cho B. Clinical usefulness of the two-site Semmes-Weinstein monofilament test for detecting diabetic peripheral neuropathy. J Korean Med Sci 2003 Feb; 18 (1): 103-7.

20. Rahman M, Griffin SJ, Rathmann W, Wareham NJ. How should peripheral neuropathy be assessed in people with diabetes in primary care? A population-based comparison of four measures. Diabetic Med 2003 May; 20 (5): 368-74.

21. McGill M, Molyneaux L, Spencer R, Heng LF, Yue DK. Possible sources of discrepancies in the use of the Semmes-Weinstein monofilament: impact on prevalence of insensate foot and workload requirements. Diabetes Care 1999 Apr; 22 (4): 598-602.

22. Börü UT, Alp R, Sargin H, Koçer A, Sargin M, Lüleci A, et al. Prevalence of peripheral neuropathy in type 2 diabetic patients attending a diabetes center in Turkey. Endocr J 2004 Dec; 51 (6): 563-7.

23. Frykberg RG, Zgonis T, Armstrong DG, Driver VR, Giurini JM, Kravitz SR, et al. Diabetic foot disorders: a clinical practice guideline (2006 revision). J Foot Ankle Surg 2006 Sep-Oct; 45 (5 Suppl): S1-66.

24. Pham H, Armstrong DG, Harvey C, Harkless LB, Giurini JM, Veves A. Screening techniques to identify people at high risk for diabetic foot ulceration: a prospective multicenter trial. Diabetes Care 2000 May; 23 (5): 606-11.

25. Tapp RJ, Shaw JE, de Courten MP, Dunstan DW, Welborn TA, Zimmet PZ; AusDiab Study Group. Foot complications in type 2 diabetes: an Australian population-based study. Diabet Med 2003 Feb; 20 (2): 10513.

26. Al-Maskari F, El-Sadig M. PrevAlence of risk factors for diabetic foot complications. BMC Fam Pract 2007 Oct 10; 8: 59.

\section{AGRADECIMENTOS}

Os autores desejam agradecer ao Núcleo de Investigação da Coordenação do Internato de Medicina Geral e Familiar da Zona Norte, pelo apoio prestado na análise dos dados, e aos seus orientadores de formação, por tudo.

\section{ENDEREÇO PARA CORRESPONDÊNCIA}

António Alberto Gonçalves Macedo

Rua D. João de Castro, 225

4435-674 Baguim do Monte

E-mail: antmac.antonio@gmail.com

Recebido em 16/09/2008

Aceite para publicação em 28/03/2010 


\section{ABSTRACT}

\section{FOOT IN RISK OF ULCERATION IN PATIENTS WITH TYPE 2 DIABETES MELLITUS}

Objectives: To determine the prevalence of foot at increased risk of ulceration in diabetic patients in a Primary Health Care population according to the classification system of the IWGDF.

Study Design: Cross-sectional analytical observational.

Location: Three Primary Care Centres (PCC) in Matosinhos, Portugal.

Population: Patients with type 2 diabetes, holders of the Diabetic Guide and residents in Portugal.

Methods: A total of 237 participants were obtained through simple random sampling technique with reposition. The data were obtained through interviews, objective examination and analysis of processes. Demographic data was collected as well as Body Mass Index, diabetes duration and glycaemic control. The presence of peripheral neuropathy and vasculopathy was assessed in order to classify patients according to the classification system of the IWGDF.

Results: Among the 237 participants, $46 \%$ were male, averaging 63 years. The prevalence of foot at increased risk of ulceration was $18.1 \%$ and it was found an association with the condition retirement.

Conclusions: This study showed that the prevalence of diabetic patients with foot at increased risk of ulceration in Primary Health Care is significant, highlighting the need for early detection of changes in the foot and investment in the education of diabetics on feet care.

Keywords: Diabetes; Diabetic foot; Primary Health Care 